\title{
Helicon wave-generated plasmas for negative ion beams for fusion
}

\author{
Ivo Furno ${ }^{l,}{ }^{*}$, Riccardo Agnello ${ }^{l}$, U. Fantz ${ }^{4}$, Alan Howling ${ }^{I}$, Remy Jacquier ${ }^{l}$, Claudio Marini ${ }^{l}$, Gennady \\ Plyushchev ${ }^{l}$, Philippe Guittienne ${ }^{2}$, and Alain Simonin ${ }^{3}$ \\ ${ }^{1}$ Ecole Polytechnique Fédérale de Lausanne (EPFL), Swiss Plasma Center, CH-1015 Lausanne, Switzerland \\ ${ }^{2}$ Helyssen, Route de la Louche 31, CH-1092 Belmont-sur-Lausanne, Switzerland \\ ${ }^{3}$ CEA, IRFM, F-13108 St Paul lez Durance, France \\ ${ }^{4}$ Max-Planck-Institut für Plasmaphysik, Boltzmannstr. 2, 85748 Garching, Germany
}

\begin{abstract}
In the next generation of fusion reactors, such as DEMO, neutral beam injectors (NBIs) of high energy $(0.8-1 \mathrm{MeV})$ deuterium atoms with high wall-plug efficiency $(>50 \%)$ will be required to reach burning plasma conditions and to provide a significant amount of current drive. The present NBI system for DEMO assumes that $50 \mathrm{MW}$ is delivered to the plasma by $3 \mathrm{NBIs}$. In the Siphore NBI concept, negative deuterium ions are extracted from a long, thin ion source $3 \mathrm{~m}$ high and $15 \mathrm{~cm}$ wide, accelerated and subsequently photo-neutralized. This requires the development of a new generation of negative ion sources. At the Swiss Plasma Center, a novel radio frequency helicon plasma source, based on a resonant network antenna source delivering up to $10 \mathrm{~kW}$ at $13.56 \mathrm{MHz}$, has been developed and is presently under study on the Resonant Antenna Ion Device (RAID). RAID is a linear device (1.9 m total length, $0.4 \mathrm{~m}$ diameter) and is equipped with an extensive set of diagnostics for full plasma characterization. In this work, the principles of operation of resonant antennas as helicon sources are introduced. We present absolute spectroscopy, Langmuir probe, and interferometry measurements on helicon plasmas. We characterize the performance of the source in terms of hydrogen/deuterium dissociation and negative ion production as a function of the input power. Furthermore, first results with the helicon birdcage antenna installed on the Cybele negative ion source at CEA-IRFM are presented, as a first step towards the validation of the Siphore concept.
\end{abstract}

\section{Introduction}

The next generation of fusion reactors, such as DEMO, will require neutral beam injectors (NBIs) of high energy $(0.8-1 \mathrm{MeV})$ deuterium atoms with high wall-plug efficiency $(>50 \%)$ to reach burning plasma conditions and provide a significant amount of current drive. The present NBI system for DEMO assumes that $50 \mathrm{MW}$ is delivered to the plasma by 3 NBIs, which can only be obtained using a negative ion-based NBI system. This has stimulated a R\&D program on negative ion production from caesiated surfaces. Recently, the use of helicon sources has been suggested as a possible candidate for plasma generation in negative hydrogen ion sources for NBIs for fusion applications [1]. For example in the Siphore concept [2], negative deuterium ions are extracted perpendicularly in a sheet-like geometry ( $3 \mathrm{~m}$ high, $15 \mathrm{~cm}$ wide) from a plasma column produced by helicon sources, accelerated and subsequently photo-neutralized.

Since the first experiments performed by Boswell [3], helicon sources have been extensively studied and they are proven to be very efficient for high-density $10^{18}$ $10^{19} \mathrm{~m}^{-3}$ plasma production (in noble gases, typically argon) with moderate injection power at low pressure.
Historically, they have found applications in semiconductor manufacturing and ion thruster systems. For fusion applications, helicon sources may have the following advantages over traditional inductivelycoupled plasma (ICP) generators: 1) reduced required radio-frequency (RF) power, leading to wider operational domain; 2) stable operation at low pressure $(<0.3 \mathrm{~Pa})$, reducing negative ion losses by electron stripping; 3) lower electron temperatures in the peripheral plasma region, resulting in higher efficiency of negative ion production in volume production mode; 4) high degree of molecular dissociation in hydrogen/deuterium plasmas, which would be favourable in a caesiated source, although disadvantageous for volume production. However, high power helicon sources are today unexplored both from the physics and technological point of view. While an extensive literature can be found on sources at low to moderate RF powers, $\sim 100 \mathrm{~W}$ to $2 \mathrm{~kW}$, there are only a few measurements at larger RF powers [4], and almost none in the $10-50 \mathrm{~kW}$ range operating in steady-state $(>0.1 \mathrm{~s})$, as required for example for the DEMO NBI system [5].

A new helicon plasma source has been recently developed at the Swiss Plasma Center of the EPFL, the

Corresponding author: ivo.furno@epfl.ch 


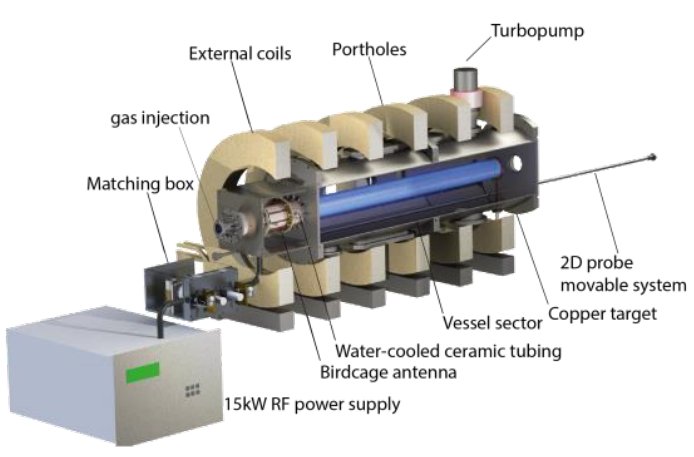

Fig. 1. Schematic cross-section of the Resonant Antenna Ion Device (RAID) with the main elements. See Fig. 2 for details of the birdcage antenna.

Resonant Antenna Ion Device (RAID). This has a planned steady-state operating power of $10 \mathrm{~kW}$ in $\mathrm{H}_{2} / \mathrm{D}_{2}$ and is currently operating in steady-state at $5 \mathrm{~kW}$. Although a single $10 \mathrm{~kW}$ helicon generator will probably not achieve the relevant plasma density required for a fusion-grade NBI source, the $10 \mathrm{~kW}$ helicon source is an intermediate step towards larger helicon powers, which will provide an opportunity to explore the main technology and physics issues related to high power, steady state helicons.

This article is organized as follows. In Sec. 2, we describe the main elements of RAID. Section 3 details the birdcage resonant antenna and its working principles. In Sec. 4, we review the first results in hydrogen and deuterium plasmas, showing that a helicon antennabased system may provide the negative ion injectors considered necessary for fusion reactor development. Future opportunities for research are outlined in Sec. 5.

\section{The Resonant Antenna Ion Device - RAID}

The Resonant Antenna Ion Device (RAID) is a flexible device allowing for full diagnostic access to study helicon wave-produced plasmas and their potential for negative ion production. Plasmas are produced and sustained using a $10 \mathrm{~kW}$ birdcage resonant network at 13.56 MHz using different gases with typical electron densities $\mathrm{n}_{\mathrm{e}} \sim 10^{18} \mathrm{~m}^{-3}$ and temperatures $\mathrm{T}_{\mathrm{e}} \sim 5 \mathrm{eV}$ in hydrogen/deuterium.

RAID has a linear vacuum chamber with radius $\mathrm{R}=0.2 \mathrm{~m}$ and length $1.5 \mathrm{~m}$. A drawing of the device with the main elements is shown in Fig. 1. The circular crosssection vacuum chamber is made of stainless-steel and is divided into two sectors. Each sector is water-cooled and possesses many port-holes of different shapes and sizes for diagnostics access. The linear sectors are installed on rails and are individually extractable, so that each sector can be easily removed to facilitate the installation of diagnostics. Spare sectors with different port-hole geometries are available and can be modified for new experiments, without stopping the experimental activity. One turbo-pump (nominal pumping speed of $345 \mathrm{l} / \mathrm{s}$ for

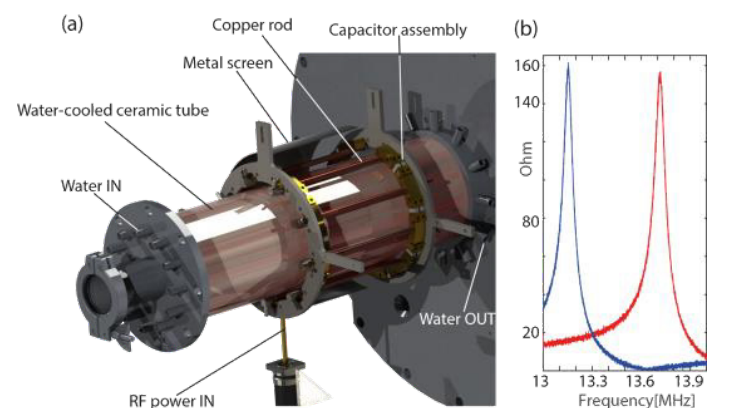

Fig. 2. (a) The birdcage resonant antenna with the different components. (b) Magnitude of the antenna impedance in vacuum for two positions of the metal screen.

nitrogen), backed by a primary pump, maintains a base pressure $<10^{-6}$ mbar inside the vessel. Gas, or a mixture of different gases ( $\mathrm{Ar}, \mathrm{He}, \mathrm{H}_{2}, \mathrm{D}_{2}, \mathrm{~N}_{2}, \mathrm{Ne}$ ) is injected either at one axial position or from the back of the helicon antenna, as shown in Fig. 1, via calibrated mass flow controller. Typical gas flow rates are $3 \mathrm{ml} / \mathrm{min}$ and 10 $\mathrm{ml} / \mathrm{min}$ in $\mathrm{H}_{2}$ and $\mathrm{Ar}$ gas, respectively, at $0.3 \mathrm{~Pa}$ base pressure. The birdcage resonant antenna is installed on the end flange of the vacuum vessel around a dielectric tube, as shown in Fig. 1. This tube consists of an assembly of two coaxial tubes (internal diameter: $9.5 \mathrm{~cm}$, external diameter: $11.5 \mathrm{~cm}$, length: $38 \mathrm{~cm}$ ) made of high thermal conductivity alumina $\left(\mathrm{Al}_{2} \mathrm{O}_{3}\right)$. The external ceramic cylinder has 8 channels for cooling the assembly by laminar water flow permitting continuous operation at high RF power. The plasma generated by the antenna forms a column of approximately constant radius terminating on a water-cooled copper plate (target), placed at the end of the chamber. The target can be grounded, floated or biased to test several types of boundary conditions.

Six water-cooled copper coils for the generation of the magnetic field surround the vacuum vessel. The spacing between adjacent coils is $30 \mathrm{~cm}$ and each coil can be independently powered up to a maximum coil current of $600 \mathrm{~A}$. This corresponds to a maximum magnetic field on-axis of $\sim 800$ Gauss when the coil currents are all in the same direction. By independently powering the magnetic coils, a variety of magnetic field configurations can be produced, including quasi-constant magnetic field, gradients and expanding magnetic field configurations.

\section{The birdcage resonant antenna}

RAID is equipped with a new type of helicon source based on the concept of a birdcage resonant antenna [6], which is shown in Fig. 2(a), using a CAD drawing. The antenna is mounted axially on one end of the vacuum chamber and is made of $\mathrm{N}=9$ conducting parallel legs distributed around a dielectric tube in a cylindrical configuration. The antenna diameter is $13 \mathrm{~cm}$ and the 15 cm-long conducting parts are made of copper tubing for water cooling of the system. Each leg is connected at both ends to its closest neighbours by capacitor assemblies. These are made of parallel arrangements of 6 high quality factor mica capacitors. The total individual 
(a) argon plasma

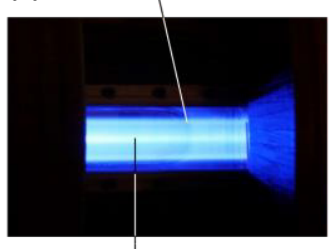

blue core (b) hydrogen plasma

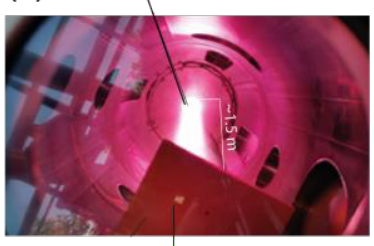

copper target

Fig. 3. (a) Lateral view of argon plasma with a clearly visible blue core indicating helicon mode ( $\sim 800$ Gauss, $0.3 \mathrm{~Pa}, 4$ $\mathrm{kW}$ ). (b) End on view of visible light emission from hydrogen plasma (150 gauss, $0.3 \mathrm{~Pa}, 3.5 \mathrm{~kW}$ ).

capacitance of each arrangement is $C=3840 \mathrm{pF}$. A review of the theory of birdcage antennas, extensively used for nuclear magnetic resonance applications, can be found in Ref. [7]. An open birdcage antenna exhibits N-1 resonant frequencies corresponding to the normal modes of the structure. When excited at one of its resonant frequencies a strong, azimuthal sinusoidal, distribution of current amplitude is generated in the antenna legs, all these currents being temporally in phase. In the neighbourhood of each resonant frequency, the antenna behaviour can be modelled in a first approximation as an L, R, C parallel resonance circuit. In the present design on RAID, a resonance of the birdcage at $13.56 \mathrm{MHz}$ is used to generate a homogenous distribution of the RF field in the centre of the structure, corresponding to a sinusoidal current distribution in the antenna legs. The choice of this frequency is dictated by the ISM standard in the industrial plasma field and the availability of RF components.

In previous studies [6], the birdcage helicon antenna was shown to be very efficient in terms of helicon wave excitation in noble gases (typically $\mathrm{Ar}, \mathrm{Xe}$, and $\mathrm{Ne}$ ). Using interferometry measurements of the electron density, a transition from inductive to helicon mode was observed in argon at relatively low injected RF power $(\sim 200 \mathrm{~W})$. Electron densities of the order of $5 \times 10^{18} \mathrm{~m}^{-3}$ were obtained in a $10 \mathrm{~cm}$ discharge tube, with argon at 1 $\mathrm{Pa}$ and for $300 \mathrm{~W}$ of power injection. It was also shown that the birdcage resonator allowed many limitations inherent to conventional antenna designs to be overcome (pressure range for which helicon regimes can be sustained, high gas flow rate of electronegative gases, non-uniform static magnetic field). An example of helicon plasma in argon in RAID is shown in Fig. 3(a). A visible light image is taken from a lateral port (perpendicular to the plasma column) and shows an intense blue core on axis (blue being the dominant ion Ar II emission), typically used as an identification of the helicon mode.

Beside its ability to efficiently excite helicon modes, the resonant network has very interesting properties in terms of its input impedance. In fact, these antennas present a large, quasi-real input impedance at the resonance, even when coupled to plasmas. This results in small electrostatic potentials of the antenna with respect to the ground thus mitigating sputtering problems and avoiding the use of Faraday shield at relatively high powers. In terms of impedance matching between the RF generator and its load it also constitutes a major

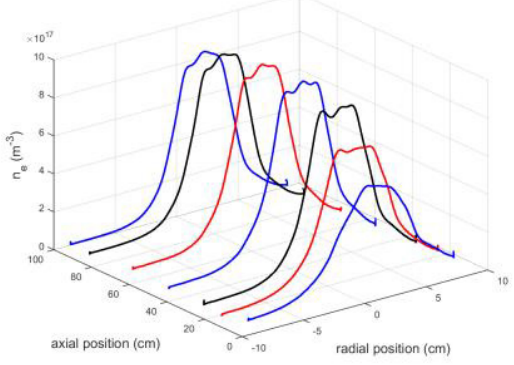

Fig. 4. Electron density profile for $\mathrm{H}_{2}$ plasma at $2 \mathrm{~kW}$. The centre of birdcage antenna is located at the axial position $\mathrm{z}=-28$

advantage with respect to conventional antennas, which have almost purely reactive impedances and are then strongly mismatched with the standard real $50 \mathrm{Ohm}$ output impedance of the RF generators. This is important to minimize power losses due to dissipation in the matching network. The electrical characterization of the RAID birdcage antenna was performed by using a commercial RF network analyzer to measure the impedance in the absence of a plasma. The magnitude of the impedance is shown in Fig. 2(b), which exhibits a clear resonance around $13.56 \mathrm{MHz}$ with a real value of approximately $160 \mathrm{Ohms}$. Similarly to flat antennas [8], during plasma operation, the position of the resonance shifts towards higher frequencies and the real impedance decreases, indicating that power is dissipated in the plasma. This frequency shift is a disadvantage when working with fixed-frequency RF power generators, since it results in an impedance mismatch condition and possibly high reflected power. Although the birdcage antenna would work even when operated slightly off its resonant frequency, the $50 \mathrm{Ohm}$ impedance matching condition at the resonance is a great advantage since it minimizes the power dissipated in the matching network. At high power, this will become of crucial importance. In RAID, the frequency shift is compensated by radially moving two semi-cylindrical metallic screens, disposed around the coil on the air side. Adjusting the screen position allows tuning the antenna resonant frequency with $2 \mathrm{MHz}$ range. The effect of the screen on the birdcage impedance is also shown in Fig. 2(b) in vacuum. Based on the measured shift in the resonant antenna frequency and the measured real impedance of the antenna with and without plasma, the power transfer efficiency from the RF antenna to the plasma is approximately $90 \%$ [8].

\section{First characterization of hydrogen and deuterium plasmas}

Here, we present first results obtained with the birdcage antenna in hydrogen and deuterium gases, which are required for neutral beam applications. In particular, we focus on conditions satisfying Siphore requirements [9] for the plasma source, namely a low base neutral pressure, $<0.3 \mathrm{~Pa}$ and magnetic field $\sim 150 \mathrm{G}$, which will provide the first step towards the validation of the Siphore concept. The discharges are steady-state with the plasma lasting for minutes at all levels of power. As an 

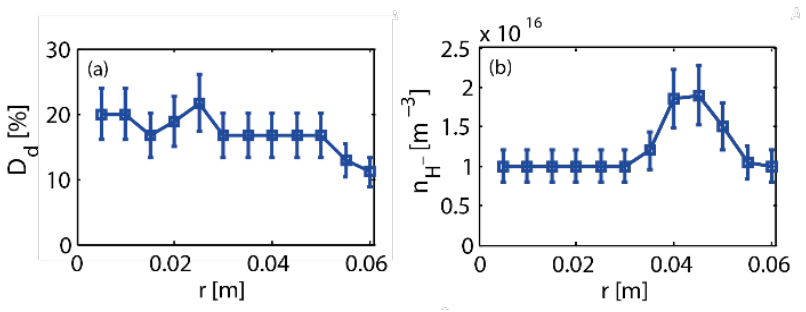

Fig. 5. Profiles of (a) the degree of dissociation $D_{d}$, and (b) the $\mathrm{H}^{-}$density estimated by YACORA for an input power of $3 \mathrm{~kW}$ in hydrogen. The presence of negative hydrogen in a shell around $r=4.5 \mathrm{~cm}$ is clearly visible in (b).

example, Fig. 3(b) shows a visible light image of a hydrogen plasma with $3.5 \mathrm{~kW}$ of injected RF power. The camera was positioned end on to provide a full axial view of the plasma column. The pressure of $0.3 \mathrm{~Pa}$, also required for operation in Siphore, is one of the source requirements for ITER and DEMO NBIs to minimize the negative ion losses due to collisions with molecules in the accelerator. As clearly seen, although the birdcage antenna is positioned behind the end flange in Fig. 3(b), the plasma column extends in the axial direction over the entire length of the vacuum vessel, consistent with a volumetric plasma production from helicon waves. The advantage of the birdcage helicon antenna, in particular its ability to produce dense and long plasma columns, with respect to the ICP driver for the Siphore ion source has already been discussed in Ref. [9]. Using a 2D system for a Langmuir probe (LP) in ion saturation mode and a $100 \mathrm{GHz}$ interferometer, we measured the profile of the electron density $n_{e}$ in a $\mathrm{H}_{2}$ plasma, shown in Fig. 4 for $2 \mathrm{~kW}$ of injected RF power. The electron density increases from $\sim 4 \times 10^{17} \mathrm{~m}^{-3}$ near the birdcage antenna to $\sim 1.1 \times 10^{18} \mathrm{~m}^{-3}$ at $30 \mathrm{~cm}$ away from it and remains approximately constant up to the end flange. This feature is particularly promising for the Siphore concept, which will require a homogenous plasma column over a $\sim 3 \mathrm{~m}$ length, to extract constant negative ion current over the entire length of the device. LP measurements (in swept mode) of the electron temperature profile are presently ongoing.

Optical emission spectroscopy (OES) measurements, detailed in Ref. [10], were interpreted using the collisional-radiative code YACORA [11] to measure the density of the six species $\mathrm{H}, \mathrm{H}^{-}, \mathrm{H}_{2}, \mathrm{H}^{+}, \mathrm{H}_{2}{ }^{+}$and $\mathrm{H}_{3}{ }^{+}$, together with the electron density $\mathrm{n}_{\mathrm{e}}$ and temperature $\mathrm{T}_{\mathrm{e}}$. Figure 5 shows the radial profiles of (a) the dissociation degree, and of (b) the $\mathrm{H}^{-}$density estimated by YACORA $68 \mathrm{~cm}$ away from the birdcage antenna in a $\mathrm{H}_{2}$ plasma with $3.5 \mathrm{~kW}$ of RF power. The dissociation degree $D_{d}=f$ $/(f+2)$ ( $f$ being the ratio of the atomic to molecular hydrogen is almost flat across the entire radius of the plasma column. The most striking feature is the presence of a radial shell between $r=3$ and $r=5 \mathrm{~cm}$, where the negative ion density reaches values of the order of $2 \times 10^{16}$ $\mathrm{m}^{-3}$. Similar OES measurements were performed in $\mathrm{H}_{2} / \mathrm{D}_{2}$ plasmas at different powers revealing that For $\mathrm{H}_{2}$, the negative ion density increases linearly with power, reaching $\sim 3 \times 10^{16} \mathrm{~m}^{-3}$ at $5 \mathrm{~kW}$, while for $\mathrm{D}_{2}$ it saturates at $\sim 4.5 \times 10^{16} \mathrm{~m}^{-3}$. These values are of interest for a negative source development operating in volume production mode.

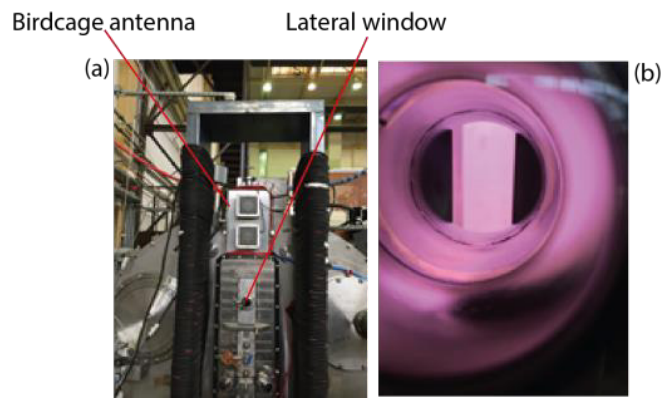

Fig. 6. (a) A picture of the antenna installed on Cybele. (b) First helicon plasma in argon in Cybele seen from a lateral window.

\section{Summary and Outlook}

The RAID device recently came online to investigate the physics and technology of high power helicon sources for NBI systems for fusion. The novel $10 \mathrm{~kW}$ birdcage resonant antenna has shown great potential for producing highly dissociated and negative ion-rich hydrogen/ deuterium plasmas, therefore opening perspectives for NBI fusion applications. A twin prototype of the helicon antenna was recently installed on the Cybele ion source [12] at the IRFM of CEA, Cadarache. Cybele is a tall and narrow ion source with a rectangular aspect ratio, which is particularly relevant to Siphore. Cybele is also equipped with a $30 \mathrm{keV}$ pre-accelerator with slotted apertures allowing to extract a thin blade-like shaped negative ion $\mathrm{H}^{-}$beam [2]. A picture of the antenna mounted on top of Cybele is shown in Fig. 6(a). Preliminary successful tests of plasma production in argon were performed up to a power of $2 \mathrm{~kW}$. An example of Ar plasma is shown in Fig. 6(b) through a lateral porthole of Cybele. Detailed optimization studies of the magnetic field are ongoing to maximize the homogeneity of the plasma along its axis. The second phase of the developments both on the RAID test bed and on Cybele will investigate the ability of the helicon source at the nominal power of $10 \mathrm{~kW}$ to produce a high negative ion density in pure volume production, and in a second step with caesium seeding on Cybele.

This work has been carried out within the framework of the EUROfusion Consortium and has received funding from the Euratom research and training programme 20142018 under grant agreement no. 633053. The views and opinions expressed herein do not necessarily reflect those of the European Commission.

\section{References}

1. U. Fantz and W. Bohm, RF workshop at $14^{\text {th }}$ ICIS (2011).

2. A. Simonin et al., New J. Phys. 18, 125005 (2016).

3. R.W. Boswell, Plasma Phys. Control. Fusion 26, 1147 (1984).

4. J. B. O. Caughman et al., Journal of Vacuum Science \& Technology A: Vacuum, Surfaces, and Films 35, 03E114 (2017); B. D. Blackwell et al., Plasma Sources Sci. Technol. 21, 55033 (2012); J. F. Caneses and B. D. Blackwell, Plasma Sources Sci. Technol. 25, 55027 
(2016).

5. P. Sonato et al., Nuc. Fusion 57, 056026 (2017).

6. P. Guittienne, E. Chevalier and Ch. Hollenstein, Journal of Applied Physics 98, 083304 (2005).

7. J. Jin, Electromagnetic analysis and designs in magnetic resonant imaging, (Boca, Raton, FL: CRC Press), 1988.

8. Ch. Hollenstein et al., Plasma Sources Science and Technology 22, 055021 (2013).

9. A. Simonin et al., Nuc. Fusion 55, 123020 (2015).

10. C. Marini et al., Nuc. Fusion 57, 036024 (2017).

11. D. Wunderlich, S. Dietrich, and U. Fantz, J. Quant. Spectrosc. Radiat. Transfer 110, 62 (2009).

12. A. Simonin and P. Garibaldi, Rev. Sci. Instrum. 12 77 03A525 (2006). 